\title{
Conocimientos sobre hidratación oral por las madres de pacientes de I a 5 años de vida con enfermedad diarreica aguda. República Dominicana
}

\author{
Lina Vásquez¹, Demian Herrera ${ }^{2}$, Mabel Segura ${ }^{2}$
}

\section{RESUMEN}

Objetivo: Determinar el nivel de conocimiento de la madre con respecto a la hidratación oral del paciente durante un episodio diarreico agudo.

Material y Métodos: Estudio observacional, descriptivo con recolección prospectiva y de corte transversal, en el cual se recolectaron datos entre la segunda semana de mayo a la segunda semana de junio 2013; con un cuestionario de 12 ítems con preguntas cerradas con el fin de medir el conocimiento de la madre con relación a la hidratación oral durante el episodio de diarrea. Se eligió la muestra de manera no probabilística por conveniencia.

Resultados: La mayoría de las madres conocía las implicancias sobre la salud de su hijo de la enfermedad diarreica aguda, y conocía la importancia de mantener una hidratación adecuada durante el mismo. Se evidenció que un porcentaje de la población, desconocía la preparación y viabilidad del Suero de rehidratación oral (SRO), y se evidenció el uso de otro tipo de bebidas no adecuadas durante el episodio de diarrea aguda. (Bebidas hidratantes comerciales y carbonatados) como terapia de hidratación durante la diarrea.

Conclusiones: Se identificó que se hace necesario una mayor información y capacitación a las madres acerca de la manera de rehidratar a sus hijos con diarrea aguda. (Horiz Med 2014; 14(3): 24-27)

Palabras clave: Diarrea, Hidratación, SRO, Bebidas hidratantes, deshidratación. (Fuente: DeCS BIREME).

\section{Knowledge about oral hydration by mothers of patients 1 to 5 years old with acute diarrhea. Dominican Republic.}

\begin{abstract}
Objective: Determine the level of Knowledge of the mother regarding oral hydration practices of a child during an acute diarrheal episode

Material and Methods: Observational, descriptive with prospective data collection and transversal cut study, in which data was collected from the second week of may until the second week of J une 2013; using a 12 item questionnaire, with close questions with the intent to measure the level of Knowledge of the mother regarding oral hydration practices of the child during an acute diarrheal episode. The simple was taken using a non-probabilistic convenience method.

Results: The maj ority of the mothers knew the health implications regarding acute diarrhea, and the importance of adequate oral hydration during the illness. However, these need to be strengthened, because it was witnessed that a percentage of the population didn't know the preparation and the viability of the ORS, and were using other non-adequate beverages during the acute diarrheal episode, like a high percentage of commercial hydration beverages and not on a high percentage the use of carbonated beverages (soda) as a hydration therapy during the diarrhea.

Conclusions: Advanced maternal age was associated with a higher rate of $\mathrm{C}$-sections and a higher risk of having a child with Down syndrome. There was no evidence that advanced maternal age is associated with increased neonatal morbidity. (Horiz Med 2014; 14(3): 24-27)
\end{abstract}

Key words: Diarrhea, Hydration, ORS, Hydration Drinks, Dehydration. (Source: MeSH NLM).

Pediatra Nutrióloga, Encargada de la Unidad De Diarrea Aguda del Hospital Infantil Dr. Robert Reid Cabral. República Dominicana.

Medico Pediatra 


\section{INTRODUCCIÓN}

La enfermedad diarreica aguda tiene una alta morbimortalidad aun con la introducción de la hidratación oral que conlleva a la prevención de trastornos hidroelectrolíticos asociados (1), disminuyendo las probabilidades de un ingreso hospitalario; continuando con determinar la causa 0 agente subyacente que permitiría instaurar un manejo etiológico $(2,3)$.

Dicha carga ha disminuido; para el 2010, se le atribuyeron 800,000 muertes en menores de 5 años (4).

Aun con la introducción de las sales de hidratación oral en 1978, luego de un brote de cólera (5); la OMS habla que es la segunda causa de muerte en menores de 5 años, afectando 2 billones de niños anualmente y de éstos, un 15 por ciento muere. Los menores de 3 años cursan con 3 episodios diarreicos por año (6).

Los sueros de hidratación oral son fáciles de preparar y administrar, son económicos y además, pueden ser ofrecidos a los pacientes en el hogar por el cuidador del paciente (7).

Al reconstituir, aportan los electrolitos necesarios y producen una recuperación más rápida y con pocos efectos adversos (8). Se le ha considerado como la intervención terapéutica más efectiva en disminuir la mortalidad por una causa (9).

Hay factores que intervienen en el uso de sales de hidratación oral por los padres, como son: el sabor del suero de hidratación oral, que no es un tratamiento etiológico del cuadro, ocasionalmente no se considera una verdadera medicina, esto hace que disminuya su uso y que en caso de usarse debido a la dificultad para que el niño lo ingiera su aporte sea menor al necesario por el mismo (10), sin embargo; el antecedente de diarrea en el hogar y los conocimientos de las implicaciones de la misma se asocia positivamente al uso de sueros de hidratación oral (9).

El uso de los sueros de hidratación oral depende de la información sobre éstos, ofrecidos por el personal de salud con relación al uso de otras medidas terapéuticas adicionales, como son los antidiarreicos y antibióticos $(10,11)$.

\section{MATERIAL Y MÉTODOS}

Este estudio es parte de un estudio mayor en el que se evaluaron los factores de riesgo asociados a la diarrea que contó con una muestra de 77 pacientes; del cual se sacó un subgrupo con la edad de 1 a 5 años que tienen una dieta similar a la de un adulto, para valorar el conocimiento de las madres sobre la hidratación durante el episodio de diarrea aguda, obteniéndose una muestra de 41 pacientes.

Estudio observacional, descriptivo con recolección prospectiva y de corte transversal, en el cual se recolectaron datos entre la segunda semana de mayo a la segunda semana de junio 2013, en la unidad de diarrea aguda del Hospital Infantil Dr. Robert Reid Cabral; utilizando un cuestionario de 12 ítems con preguntas cerradas con el fin de medir el comportamiento de los padres con relación a la hidratación durante el episodio de diarrea.

Se eligió la muestra de manera no probabilística por conveniencia a los pacientes que tuvieron los criterios del estudio; del cual se excluyeron:

- pacientes con enfermedad infecciosa sobre agregada que pueda provocar una enfermedad diarreica aguda.

- patología de base que predisponga a la enfermedad diarreica aguda.

- patología de base que en su sintomatología se encuentra la diarrea.

- pacientes con vómitos asociados a la diarrea.

Se utilizó para el análisis estadístico el programa Microsoft Excel, donde se generó la base de datos que fue analizada utilizando el paquete estadístico SPSS 22, donde se analizó el comportamiento individual de las variables y se valoró su significancia estadística.

\section{RESULTADOS}

Se obtuvo un total de 41 pacientes, de los cuales el $51.2 \%$ fueron de sexo femenino. 
La mayoría tuvo edad entre 12 a 24 meses de vida (46.3\% de la muestra). La frecuencia del episodio fue decreciendo a medida que aumentaba la edad (Fig. 1).

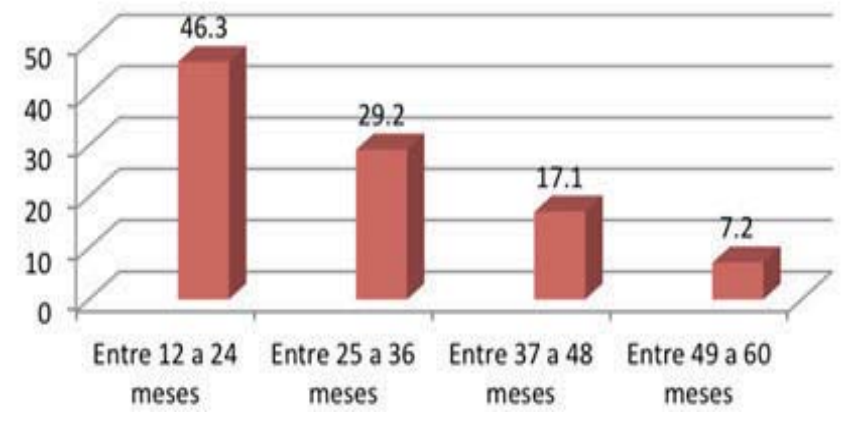

Figura 1. Distribución por grupos de edad de los pacientes

Con respecto a la edad materna, el grupo de edad mas frecuente fue de 21 a 25 , seguido por el de 26 a 30 con igual proporción de madres, con un $31.7 \%$ (Fig. 2).

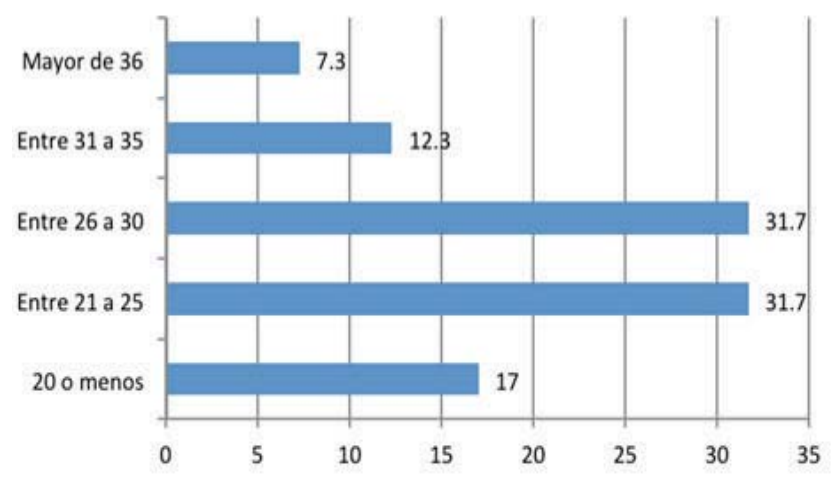

Figura 2. Indicación de cesárea.

El 43.9\% de las madres tenía escolaridad primaria, mientras que solo un $12.2 \%$ tenía una escolaridad universitaria (Fig. 3).

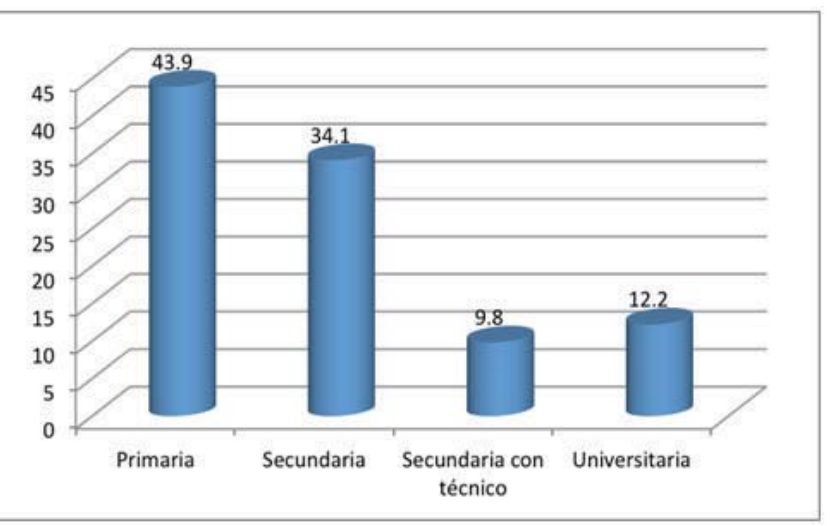

Figura 3. Grado de escolaridad de la madre
Sobre los conocimientos de hidratación oral, un 61\% de las madres, desde que empieza el episodio de diarrea empiezan a aumentar el aporte hídrico a sus hijos. De éstas, un $87.8 \%$ refleja que aumenta mayormente la cantidad de agua ofrecida. Sólo 65.9 por ciento de las madres utilizó suero de rehidratación oral (SRO) como terapia de hidratación oral en este episodio de enfermedad diarreica aguda.

El $65.9 \%$ de las madres fueron informadas por un personal de salud sobre el uso de SRO durante el episodio de diarrea aguda. Un $97.6 \%$ de las madres sabía que la enfermedad diarreica puede ocasionar deshidratación y un $90.2 \%$ conocen que dicha patología puede causar la muerte del niño.

Sólo un $75.6 \%$ de las madres sabían como preparar la sal de hidratación oral, mientras que sólo un $61 \%$ sabía el tiempo de viabilidad de la misma luego de ser reconstituida.

Un 51.2\% usó normalmente SRO para la hidratación durante un episodio de diarrea aguda, mientras que un $48.7 \%$ refirió que usaba agua; otro tipo de bebidas fueron las bebidas hidratantes comerciales con un $39 \%$.

\section{DISCUSIÓN}

Sobre la orientación del uso de los sueros de hidratación oral como terapia durante el episodio de diarrea aguda, en nuestro estudio un $65.8 \%$ de las madres recibió información por un personal de salud sobre su uso mientras que en el estudio de Mengistie et al (7) fue de $78.9 \%$. Evidenciamos un mayor uso de las SRO como terapia durante el episodio en nuestro estudio que en el de Mengistie et al (7): $65.8 \%$ versus $57.8 \%$, y superior al estudio de Gao et al (8) que tuvo un $34.6 \%$. En el estudio de Olson et al (9) el porcentaje de la población que ofreció SRO fue de un $45 \%$ y un $41 \%$ ofreció otro tipo de bebidas orales.

Cuando se preguntó sobre qué tipo de bebidas normalmente utilizaban, el porcentaje disminuyó de un $65.8 \%$ (que fue el porcentaje de uso de SRO, durante este episodio) a un $51.3 \%$, que es el porcentaje con que mayormente usaban dicha terapia de hidratación oral, un porcentaje menor 
al de Mengistie et al (7); el uso de SRO y de agua potable fueron métodos más usados de manera sola o mixta pero nunca ambos combinados. Entre otras terapias de hidratación que adicional al agua o SRO se utilizó, fue el uso de bebidas hidratantes comerciales, por lo cual cabe resaltar que hay que concientizar a la población de su uso en la enfermedad diarreica aguda ya que evidenciamos que un $39 \%$ de las madres se la ofrecen a sus hijos como terapia. Otra bebida fueron los jugos, en un $36.5 \%$ de la población.

Sobre el conocimiento de las complicaciones y la deshidratación tanto en nuestro estudio como en el de Olson et al (9), el porcentaje superó un 95 por ciento, sin embargo; las prácticas y conocimientos de las madres no fueron reflejadas con relación a las medidas realizadas durante la enfermedad.

Aunque un porcentaje elevado de las madres ofrecían SRO durante el episodio de diarrea aguda en comparación con los demás estudios, cabe destacar que un $24.6 \%$ de las mismas no sabían preparar el SRO y un $39 \%$ no conocía el tiempo de viabilidad del mismo, así que aunque se utilice no significa que sea de manera adecuada.

En conclusión, la mayoría de las madres conocía las implicancias sobre la salud de su hijo de la enfermedad diarreica aguda, y conocía sobre la importancia de mantener una hidratación adecuada durante el mismo. Sin embargo, debe reforzarse dicha medida, dado que se evidenció que no todas las madres conocen la preparación y viabilidad del SRO, y se evidenció el uso de otro tipo de bebidas no adecuadas como: bebidas hidratantes comerciales y carbonatadas.

\section{Fuentes de financiamiento}

El estudio ha sido autofinanciado por el autor.

\section{Conflicto de interés}

El autor declara no tener ningún conflicto de interés.

Recibido: 13 de Mayo de 2014 Aprobado: 12 de Agosto de 2014

\section{REFERENCIAS BIBLIOGRÁFICAS}

1. Hossein S, Omid Y, Mohammadreza M, Narges R. Lactose-Free Compared with Lactose-Containing Formula in Dietary Management of Acute Childhood Diarrhea. Iran J Pediatr Mar 2012; 22 (1): 82-86.

2. Patro B, Golicki D, Szajewska H. Meta-analysis: zinc supplementation for acute gastroenteritis in children. Alimentary Pharmacology \& Therapeutics 2008; 28(6): 713-723.

3. Long K, Rosado J, Montoya Y, Solano M, Hertzmark E, Dupont $\mathrm{H}$, Santos J. Effect of Vitamin A and Zinc Supplementation on Gastrointestinal Parasitic Infections Among Mexican Children. Pediatrics 2007; 120: E846. DOI: 10.1542/peds.2006-2187

4. Michelle G, Kerri W, Diego B, Zulfiqar B. Dietary management of childhood diarrea in low- and middle-income countries: a systematic review. BMC Public Health 2013, 13 (Suppl 3):S17.http://www. biomedcentral.com/1471-2458/13/S3/S17

5. Ciccarelli S, Stolfi I, Caramia G. Management strategies in the treatment of neonatal and pediatric gastroenteritis. Infect Drug Resist. 2013, 6: 133-161.

6. Brian A Maponga, Daniel Chirundu, Notion T Gombe, Mufuta Tshimanga, Gerald Shambira, Lucia Takundwa Risk factors for contracting watery diarrhoea in Kadoma City, Zimbabwe, 2011: a case control study. BMC infectious Diseases 2013; 13:567.

7. Mengistie B, Berhane Y, Worku A. Predictors of Oral Rehydration Therapy use among under-five children with diarrhea in Eastern Ethiopia: a community based case control study. BMC Public Health 2012; 12:1029.

8. Wenlong G, Hong Y, Duolao W, Shaonong D. Oral rehydration salt use and its correlates in low-level care of diarrhea among children under 36 months old in rural Western China. BMC Public Health. 2013; 13: 238.

9. Olson CK1, Blum LS, Patel KN, Oria PA, Feikin DR, Laserson KF, Wamae AW, Bartlett AV, Breiman RF, Ram PK. Community Case Management of Childhood Diarrhea in a Setting with Declining Use of Oral Rehydration Therapy: Findings from Cross-Sectional Studies among Primary Household Caregivers, Kenya. Am J Trop Med Hyg 2011 Dec; 85(6):1134-40.

10. Zwisler G, Simpson E, Melissa M. Treatment of diarrhea in young children: results from surveys on the perception and use of oral rehydration solutions, antibiotics, and other therapies in India and Kenya. J Glob Health 2013; 3(1): 104.

11. Arvelo W, Degollado J, Reyes L, Álvarez A. Perceptions regarding oral rehydration solutions for the management of diarrhea in Guatemalan children: implications for diarrheal management in the Americas. Rev Panam Salud Pública 2013 Aug; 34(2):121-6.

\section{Correspondencia:}

Demian Herrera Morban

Dirección: Ensanche La Paz, Santo Domingo, Distrito Nacional. República Dominicana

Teléfono: + 8297121824

Correo electrónico: herreramorbanmd@gmail.com 